Perinatology pISSN 2508-4887 • elSSN 2508-4895

\title{
Risk Factors and Neonatal Outcomes of Patent Ductus Arteriosus Ligation in Preterm Infants in a Single Center over 6 Years
}

\section{Ga Won Jeon, MD, PhD}

Department of Pediatrics, Inje University Busan Paik Hospital, Inje University College of Medicine, Busan, Korea

Received: 15 July 2020

Revised: 28 August 2020

Accepted: 21 October 2020

Correspondence to

Ga Won Jeon, MD, PhD

Department of Pediatrics, Inje

University Busan Paik Hospital, Inje University College of Medicine, 75

Bokji-ro, Busanjin-gu, Busan 47392, Korea

Tel: +82-51-890-6497

Fax: $+82-51-890-5830$

E-mail: iamgawon@hanmail.net

Copyright@ 2020 by The Korean Society of Perinatology

This is an Open Access article distributed under the terms of the Creative Commons Attribution Non-Commercial License (http://creativecommons.org/ license/by-nc/4.0/), which permits unrestricted non-commercial use, distribution, and reproduction in any medium, provided that the original work is properly cited.
Objective: To determine the effects of patent ductus arteriosus (PDA) ligation, clinical characteristics of infants who need surgical ligation of PDA and risk factors of PDA ligation were evaluated.

Methods: Preterm infants with gestational age (GA) $<30$ weeks presenting with hemodynamically significant ductus arteriosus (HSDA) were enrolled. HSDA was closed with ibuprofen (medical group, $n=55$ ) or surgical ligation (surgical group, $n=57$ ).

Results: GA and birth weight were smaller in the surgical group (27.8 \pm 1.4 weeks in the medical group vs. $26.1 \pm 1.6$ weeks in the surgical group, $P<0.001 ; 959 \pm 241 \mathrm{~g}$ in the medical group vs. $815 \pm 193 \mathrm{~g}$ in the surgical group, $P=0.001$, respectively). There were no significant differences in gender, Apgar scores, antenatal steroids therapy, maternal gestational diabetes mellitus, pregnancy-induced hypertension, or chorioamnionitis between the two groups. In the surgical group, total duration of mechanical ventilation and duration of invasive ventilation were longer, although postnatal day of ductal closure was not delayed. Moderate to severe bronchopulmonary dysplasia, high-grade intraventricular hemorrhage ( $\geq$ grade 3 ), retinopathy of prematurity ( $\geq$ stage 2 ), necrotizing enterocolitis ( $\geq$ stage 2), and mortality rate were also similar between the two groups. Low GA was a significant risk factor associated with surgical ligation of PDA (adjusted odds ratio $0.493,95 \%$ confidence interval 0.366 0.666; $P<0.001)$.

Conclusion: Although surgical ligation of PDA is inevitable in certain cases with large shunt and severe respiratory failure, clinicians should weigh the benefit of ductal closure and complications associated with surgery.

Keywords: Bronchopulmonary dysplasia, Ibuprofen, Ligation, Patent ductus arteriosus, Preterm infants

\section{Introduction}

The ductus arteriosus is a crucial fetal circulatory pathway. It closes functionally by three days after birth in healthy term infants and then anatomically closes completely. Failure of postnatal closure of the ductus arteriosus is referred to as patent ductus arteriosus (PDA). PDA is one of the most common heart problems in preterm infants. It is inversely proportional to gestational age (GA). PDA is detected up to $40-60 \%$ of preterm infants with GA $<29$ weeks, $70 \%$ of preterm infants with GA $<28$ weeks, and $80 \%$ of preterm infants with GA of 24 25 weeks. ${ }^{1,2}$ PDA in preterm infants is historically known to be associated with increased mortality and acute or chronic morbidities such as bronchopulmonary dysplasia (BPD), necrotizing enterocolitis (NEC), intraventricular hemorrhage (IVH), and retinopathy of prematurity (ROP). ${ }^{3}$ To improve survival and outcomes of preterm infants, PDA is closed mandatory with medical or surgical treatment. Prophylactic indomethacin administration soon after birth has been used to close PDA immediately after birth to improve outcomes. ${ }^{4,5}$ However, recent studies have indicated that prophylactic indomethacin is not associated 
with decreased mortality or BPD based on systemic reviews and meta-analyses. ${ }^{6,7}$ Thus, prophylactic indomethacin is not used routinely in many units, although there are unit variations of treatment. PDA in preterm infants is one of the most controversial topics in neonatology. There is still no consensus about which PDA has to be treated, when to be treated, or when to be ligated. Surgical ligation of PDA is sometimes inevitable in certain huge PDA with severe cardiorespiratory symptoms, especially in extreme preterm infants, though there are perioperative or anesthetic risks. IVH and serious pulmonary hemorrhage related to hemodynamic changes of PDA at early postnatal days can affect early postnatal mortality and neurodevelopmental impairment. ${ }^{8}$ However, there is no standard guideline about which PDA has to be ligated or when to be ligated in preterm PDA. Thus, the objective of this study was to evaluate clinical characteristics of patients who needed surgical ligation and determine risk factors and outcomes of surgical ligation of PDA.

\section{Methods}

\section{Study population}

This study was reviewed and approved by the Institutional Review Board of Inje University Busan Paik Hospital (identification code: 20-0081) in accordance with the Declaration of Helsinki. The Institutional Review Board waived the need for informed consent due to its retrospective nature. Medical records of 168 preterm infants with GA $<30$ weeks presenting with PDA who were admitted to Inje University Busan Paik Hospital from January 2014 to December 2019 were reviewed. Preterm infants with chromosomal abnormalities or life-threatening major congenital anomalies such as complex heart anomalies and pulmonary hypoplasia were excluded. Of 168 enrolled infants, 134 presented with hemodynamically significant ductus arteriosus (HSDA). The remaining 34 infants had non-HSDA. Of 134 infants with HSDA, 22 infants were excluded because their ductal closure was not confirmed after the diagnosis of PDA due to death (19 infants [12 sepsis, 5 high grade IVH, 1 severe intrauterine growth restriction with multi-organ failure, 1 spontaneous bowel perforation]) or transfer (3 infants [1 NEC, 2 high grade $[V H]$ ). Among these 22 infants, two were not treated with ibuprofen, 15 infants were treated with 1 cycle of ibuprofen, and 5 infants with 2 cycles of ibuprofen.

For 55 infants, PDA was closed with medical treatment (medical group, n=55). For 57 infants, surgical ligation of PDA was performed (surgical group, n=57) (Fig. 1).

\section{Study design}

Echocardiography (VividS5; GE Healthcare, Chicago, IL, USA) was performed within the first week of life. HSDA was defined as a predominant left to right ductal shunt flow with a diameter of $2 \mathrm{~mm}$ or more by echocardiography with hypotension, widened pulse pressure, deterioration in respiratory symptoms and requirement of respiratory support. ${ }^{9} \mathrm{HSDA}$ was treated with ibuprofen. Echocardiography was repeated in one week of ibuprofen treatment.

Intravenous ibuprofen was administered at an initial dose of $10 \mathrm{mg} / \mathrm{kg}$ followed by a $5 \mathrm{mg} / \mathrm{kg}$ after 24 hours and a second $5 \mathrm{mg} / \mathrm{kg}$ after 48 hours. Surgical ligation was performed if ibuprofen treatment was contraindicated or if HSDA failed to close with 1 or 2 cycles of ibuprofen. The attending physician decided the ligation of HSDA based on failure of medical treat-

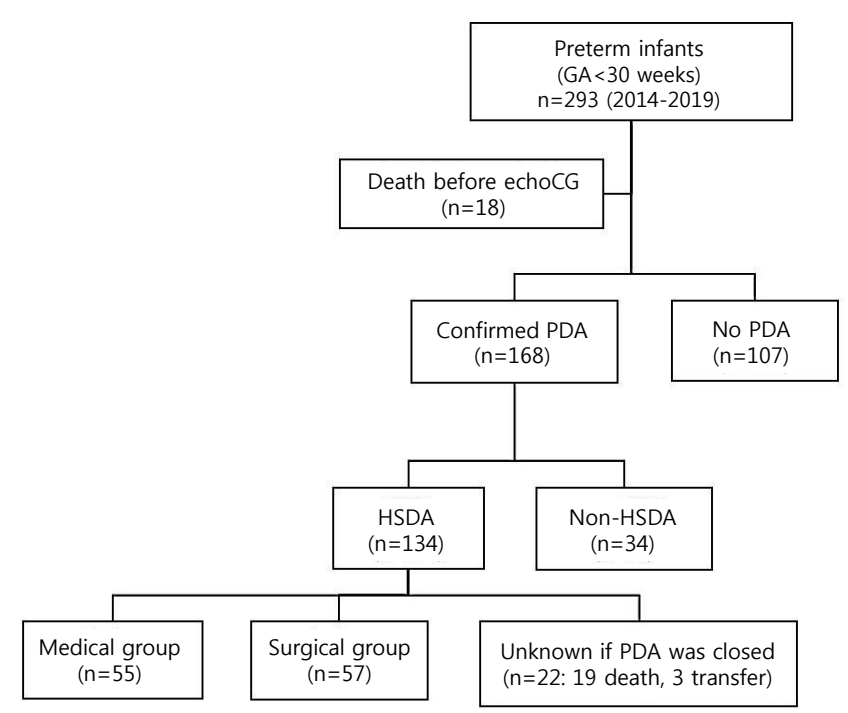

Fig. 1. Flow chart showing the selection of patients. Among 293 preterm infants with gestational age (GA) $<30$ weeks (January 2014 to December 2019), 168 infants were confirmed to have patent ductus arteriosus (PDA). Among 168 infants with PDA, 134 infants presented hemodynamically significant ductus arteriosus (HSDA). PDAs in the medical group $(n=55)$ were closed medically. PDAs in the surgical group $(n=57)$ were closed by surgical ligation. echoCG, echocardiography. 
ment or existence of a contraindication for medical treatment.

Contraindications to ibuprofen treatment included bleeding tendency, thrombocytopenia (platelet count $<50 \times 10^{3} / \mu \mathrm{L}$ ), high-grade IVH ( $\geq$ grade 3 ), decreased urination (urine output $<0.6 \mathrm{~mL} / \mathrm{kg} / \mathrm{hr})$, elevated serum creatinine level $(>2.0 \mathrm{mg} / \mathrm{dL})$, and $\operatorname{NEC}(\geq$ stage 2$){ }^{9}$ Demographic factors included GA, birth weight, gender, Apgar score, respiratory distress syndrome (RDS), surfactant re-dosing, antenatal steroids therapy, maternal gestational diabetes mellitus (GDM), maternal pregnancyinduced hypertension (PIH), and histologically confirmed chorioamnionitis to compare medical group with surgical group. Outcomes included total duration of mechanical ventilation, duration of invasive ventilation (conventional ventilation+high frequency ventilation), duration of high frequency ventilation, duration of hospital stay, BPD (moderate to severe), high-grade IVH ( $\geq$ grade 3$),{ }^{10}$ high-stage ROP ( $\geq$ stage 2$),{ }^{11} \mathrm{NEC} \mathrm{(} \geq$ stage $2),{ }^{12}$ and mortality rate to compare medical group with surgical group. Postnatal day of ductal closure was defined as the postnatal day of confirmed ductal closure by echocardiography in the medical group, or operation day in the surgical group.

Surfactant can be administered as a prophylactic therapy in infants with GA <30 weeks according to notification No. 2010135 from the Ministry of Health and Welfare in January $2011 .{ }^{13}$ Thus, surfactant was administered to all enrolled patients. The attending physician decided surfactant re-dosing based on patient severity determined by chest radiography, blood gas analysis, and ventilator settings with need for $>40 \%$ oxygen. BPD was defined as an oxygen dependency at postmenstrual age 36 weeks with oxygen treatment for at least 28 days of life. It was categorized into three groups by severity: mild, moderate, and severe BPD. ${ }^{14}$

\section{Statistical Analysis}

All statistical analyses were performed using $t$-test for continuous variables with a normal distribution and homogeneous variance. Mann-Whitney $U$-test was used for variables without a normal distribution or without homogeneous variance, such as GA. Chi-square test or Fisher's exact test was performed for nominal variables.

To assess risk factors of surgical ligation, GA and birth weight were adjusted, and the analysis of covariance was performed for continuous variables such as Apgar scores, duration of me- chanical ventilation, and hospital stay. Multivariate logistic regression analysis was performed for nominal variables such as antenatal steroids therapy, maternal GDM, maternal PIH, and chorioamnionitis. Odds ratio (OR) and 95\% confidence interval (CI) were calculated.

$P$-values $<0.05$ were considered significant. Data are given as mean \pm standard deviation. All statistical analyses were performed using IBMSPSS version 25.0 (IBM Corp., Armonk, NY, USA).

\section{Results}

\section{Infant and maternal demographic factors}

GA was younger in the surgical group compared to the medical group (27.8 \pm 1.4 weeks in the medical group vs. $26.1 \pm 1.6$ weeks in the surgical group, $P<0.001$ ). Birth weight was smaller in the surgical group compared to the medical group $(959 \pm 241 \mathrm{~g}$ in the medical group vs. $815 \pm 193 \mathrm{~g}$ in the surgical group, $P=0.001$ ). There were no significant differences in gender, Apgar score at 1 or 5 minutes, RDS, or the need for surfactant re-dosing (7 [12.7\%] in the medical group vs. 9 [15.8\%] in the surgical group, $P=0.643$, adjusted $P$ for GA and birth weight=0.125) between the two groups. There were no significant differences in antenatal steroids therapy, maternal GDM, maternal PIH, or chorioamnionitis between the two groups (Table 1).

Surgical ligation was performed because ibuprofen treatment was contraindicated in two infants (at 8th and 11th postnatal day); HSDA failed to close with 1 cycle of ibuprofen in 12 infants; and HSDA failed to close with 2 cycles of ibuprofen in 43 infants among 57 infants in the surgical group. HSDA was closed with 1 cycle of ibuprofen in 44 infants, and 2 cycles of ibuprofen in 11 infants among 55 infants in the medical group.

\section{Morbidity and outcomes}

Total duration of mechanical ventilation (32.3 \pm 23.2 days in the medical group vs. $59.4 \pm 41.3$ days in the surgical group, $P<0.001$, adjusted $P$ for $\mathrm{GA}$ and birth weight=0.029) and duration of invasive ventilation (20.1 \pm 18.4 days in the medical group vs. 42.5 \pm 28.4 days in the surgical group, $P<0.001$, adjusted $P$ for GA and birth weight $=0.004$ ) were longer in the surgical group compared to the medical group. Duration of high frequency ventilation was longer in the surgical group (4.5 \pm 7.6 days in the 
Table 1. Infant and Maternal Demographic Factors

\begin{tabular}{|c|c|c|c|c|}
\hline & Medical group $(n=55)$ & Surgical group $(n=57)$ & $P$-value & $P$-value ${ }^{*}$ \\
\hline Gestational age (weeks) & $27.8 \pm 1.4$ & $26.1 \pm 1.6$ & $<0.001^{\dagger}$ & - \\
\hline Birth weight (g) & $959 \pm 241$ & $815 \pm 193$ & $0.001^{\ddagger}$ & - \\
\hline Gender (male) & $28(50.9)$ & $28(49.1)$ & 0.850 & $0.983^{\phi}$ \\
\hline Apgar score at 1 minute & $4.2 \pm 1.3$ & $4.1 \pm 1.3$ & $0.756^{\ddagger}$ & $0.054^{\| \prime}$ \\
\hline Apgar score at 5 minutes & $6.8 \pm 0.9$ & $6.5 \pm 1.1$ & $0.123^{\ddagger}$ & $0.753^{11}$ \\
\hline RDS & $55(100.0)$ & $57(100.0)$ & $>0.999^{\natural}$ & $>0.999^{\phi}$ \\
\hline Surfactant re-dosing & $7(12.7)$ & $9(15.8)$ & $0.643^{n}$ & $0.125^{\phi}$ \\
\hline Antenatal steroids therapy & 49 (89.1) & $53(93.0)$ & $0.470^{\prime \prime}$ & $0.800^{6}$ \\
\hline GDM & $2(3.6)$ & $2(3.5)$ & $0.971^{* *}$ & $0.906^{\phi}$ \\
\hline $\mathrm{PIH}$ & $7(12.7)$ & $7(12.3)$ & $0.943^{n}$ & $0.320^{\oint}$ \\
\hline Chorioamnionitis & $25 / 54(46.3)$ & $31 / 52(59.6)$ & $0.170^{\prime \prime}$ & $0.184^{\oint}$ \\
\hline
\end{tabular}

Values are presented as mean \pm standard deviation or number (\%).

Abbreviations: RDS, respiratory distress syndrome; GDM, gestational diabetes mellitus; PIH, pregnancy induced hypertension.

${ }^{*}$ Adjusted for gestational age and birth weight.

${ }^{\dagger}$ Mann-Whitney U-test.

${ }^{\ddagger} t$-test.

${ }^{\$}$ Multivariate logistic regression analysis.

"Analysis of covariance.

"Chi-square test.

${ }^{* *}$ Fisher's exact test.

medical group vs. 8.1 \pm 11.2 days in the surgical group, $P=0.049$ ). However, it became similar after adjusting for GA and birth weight (adjusted $P$ for GA and birth weight=0.574). Duration of hospital stay was longer in the surgical group (79.3 \pm 33.8 days in the medical group vs. $105.2 \pm 44.8$ days in the surgical group, $P=0.001$ ). However, it was similar between the two groups after adjusting for GA and birth weight (adjusted $P$ for GA and birth weight $=0.114$ ).

Cases of moderate to severe BPD (21.7\% in the medical group vs. $36.7 \%$ in the surgical group, $P=0.109$, adjusted $P$ for GA and birth weight $=0.831)$ and ROP ( $\geq$ stage 2$)(35.3 \%$ in the medical group vs. $50.0 \%$ in the surgical group, $P=0.128$, adjusted $P$ for GA and birth weight $=0.796)$ were similar between the two groups. Cases of IVH ( $\geq$ grade 3 ), NEC ( $\geq$ stage 2 ), and mortality rate were also similar between the two groups. Postnatal day of ductal closure was also similar between the two groups (24.0 \pm 29.6 days in the medical group vs. $20.3 \pm 13.2$ days in the surgical group, $P=0.390$, adjusted $P$ for $G A$ and birth weight $=0.948$ ) (Table 2).

\section{Risk factors of surgical ligation}

Low GA was a significant risk factor associated with surgical ligation of PDA based on logistic regression analysis (adjusted
OR 0.493, 95\% CI 0.366-0.666; $P<0.001)$. Low birth weight (adjusted OR 1.000, 95\% CI 0.997-1.003; $P=0.978$ ), low Apgar score (adjusted OR 1.354, 95\% CI 0.916-2.001, $P=0.128$ for Apgar score at 1 minute; adjusted OR 0.687, 95\% CI 0.3571.324, $P=0.262$ for Apgar score at 5 minutes), antenatal steroids therapy (adjusted OR 0.999, 95\% CI 0.208-4.789; $P=0.999$ ), maternal GDM (adjusted OR 0.573, 95\% CI 0.042-7.763; $P=$ 0.675), maternal PIH (adjusted OR 0.511, 95\% CI 0.103-2.534; $P=0.411$ ), and chorioamnionitis (adjusted OR 1.908, 95\% CI 0.689-5.285; $P=0.214$ ) were not risk factors associated with surgical ligation of PDA (Fig. 2).

\section{Discussion}

Preterm infants, especially extreme preterm infants, have a high risk of IVH and serious pulmonary hemorrhage related to hemodynamic changes of HSDA at early postnatal days. This might affect long-term neurodevelopmental impairment as well as early postnatal mortality. ${ }^{8}$ Also, treatment-related complications must be considered as well as the risk of having PDA. To minimize complications of PDA itself and complications of treatment, which PDA has to be treated, when to be treated, and 
Table 2. Morbidity and Outcomes

\begin{tabular}{|c|c|c|c|c|}
\hline & Medical group $(n=55)$ & Surgical group $(n=57)$ & $P$-value & $P$-value \\
\hline Total duration of mechanical ventilation (days) & $32.3 \pm 23.2$ & $59.4 \pm 41.3$ & $<0.001^{\dagger}$ & $0.029^{\ddagger}$ \\
\hline Duration of invasive ventilation (days) & $20.1 \pm 18.4$ & $42.5 \pm 28.4$ & $<0.001^{\dagger}$ & $0.004^{\ddagger}$ \\
\hline Duration of high frequency ventilation (days) & $4.5 \pm 7.6$ & $8.1 \pm 11.2$ & $0.049^{\dagger}$ & $0.574^{\ddagger}$ \\
\hline Hospital stay (days) & $79.3 \pm 33.8$ & $105.2 \pm 44.8$ & $0.001^{\dagger}$ & $0.114^{\ddagger}$ \\
\hline BPD (moderate to severe) & $10 / 46(21.7)$ & $18 / 49(36.7)$ & $0.109^{\oint}$ & $0.831^{\prime \prime}$ \\
\hline IVH ( $\geq$ grade 3 ) & $9(16.4)$ & $12(21.1)$ & $0.525^{\text {f }}$ & $0.523^{11}$ \\
\hline ROP ( $\geq$ stage 2 ) & $18(35.3)$ & $27 / 54(50.0)$ & $0.128^{\phi}$ & $0.796^{11}$ \\
\hline NEC ( $\geq$ stage 2 ) & $9(16.4)$ & $5(8.8)$ & $0.225^{\oint}$ & $0.168^{\| \prime}$ \\
\hline Mortality rate & $8(14.5)$ & $9(15.8)$ & $0.854^{6}$ & $0.836^{\| \prime}$ \\
\hline PDA closure (postnatal day) & $24.0 \pm 29.6$ & $20.3 \pm 13.2$ & $0.390^{\dagger}$ & $0.948^{\ddagger}$ \\
\hline
\end{tabular}

Values are presented as mean \pm standard deviation or number (\%).

Abbreviations: BPD, bronchopulmonary dysplasia; IVH, intraventricular hemorrhage; ROP, retinopathy of prematurity; NEC, necrotizing enterocolitis; PDA, patent ductus arteriosus.

${ }^{*}$ Adjusted for gestational age and birth weight.

${ }^{\dagger}$ t-test.

${ }^{\ddagger}$ Analysis of covariance.

${ }^{\S}$ Chi-square test.

"Multivariate logistic regression analysis.

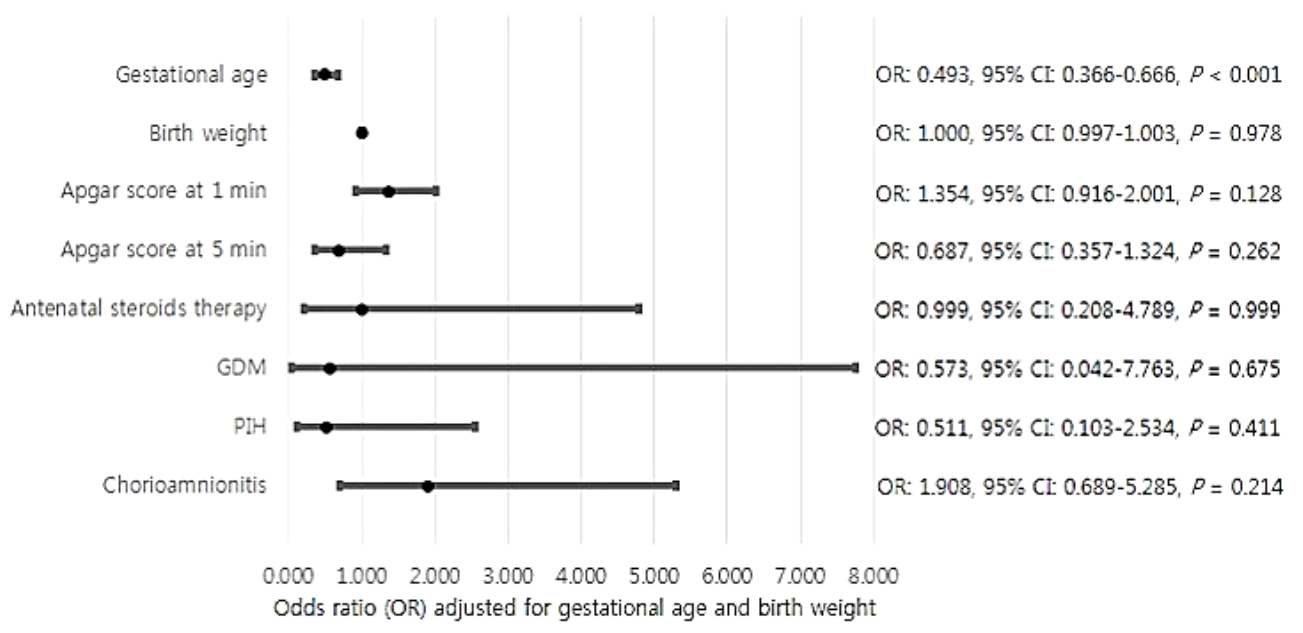

Fig. 2. Risk factors of surgical ligation. Low gestational age (GA) was a significant risk factor associated with surgical ligation of patent ductus arteriosus (PDA) based on logistic regression analysis (adjusted odds ratio [OR] 0.493, 95\% confidence interval [Cl] 0.366-0.666, $P<0.001)$. GDM, gestational diabetes mellitus; $\mathrm{PIH}$, pregnancy induced hypertension.

when to be ligated are critically important, especially for extreme preterm infants. Optimal timing of surgical ligation is also important, especially for those with huge PDA and severe cardiorespiratory symptoms.

Surgical ligation is usually performed when medical treatment has failure or contraindication. Thus, infants who need surgical ligation after failure of medical treatment tend to be exposed to PDA longer than infants whose PDA is successfully closed with medical treatment. Prolonged PDA increases hydraulic pressures on the pulmonary capillary bed, leading to capillary endothelial injury and increased fluid filtration into the interstitium. Finally, it may result in pulmonary edema, the development of BPD, and prolonged ventilator support. ${ }^{15} \mathrm{How}^{-}$ ever, in our previous study, delayed ductal closure (PDA closure $\geq$ postnatal 14 days) was safe, and did not increase mortality or BPD. ${ }^{16}$ Caregivers have to take a careful consideration of 
medical or surgical treatment during early postnatal period in terms of complications of treatment. ${ }^{16}$

In this study, the duration of exposure to PDA was not prolonged in the surgical group. Nonetheless, the surgical group needed longer ventilator support, especially longer invasive ventilator support. Thus, there must be factors other than PDA shunt flow, which affect pulmonary condition and subsequent ventilator support as well as the development of BPD in the surgical group. Various peri-operative and anesthetic complications such as hemothorax, chylothorax, pneumothorax, nerve injury, infection, bleeding, volume overloading, and abrupt hemodynamic changes can happen during PDA ligation. Various peri-operative and anesthetic complications might be related to pulmonary over-circulation, heart failure, and BPD.

The value of surgical ligation of HSDA is still controversial. According to Ezenwa et al., ${ }^{17}$ PDA ligation could not improve mortality, BPD, severe IVH, NEC, or neurodevelopmental outcomes in extremely preterm infants. Moreover, surgical treatment of PDA has been reported to be associated with BPD, ROP, and neurodevelopmental impairment. ${ }^{18,19}$ On the other hand, surgical treatment of PDA has been reported to be associated with decreased mortality without increasing BPD, ROP, or neurodevelopmental impairment. ${ }^{20,21}$ Rather, surgical treatment of PDA was associated with decreased mortality and increased neurodevelopmental impairment in survivors. ${ }^{22}$ Surgical treatment is recommended if there are suboptimal cerebral perfusion and subsequent suboptimal cerebral oxygenation caused by PDA. This is because suboptimal cerebral perfusion and oxygenation may result in decreased brain volume and neurodevelopmental impairment. ${ }^{23}$ According to Weisz and Giesinger, ${ }^{24}$ neurodevelopmental impairment is not associated with surgical treatment of PDA itself. Weisz and Giesinger ${ }^{24}$ have suggested a selection bias as infants with high-grade IVH are more likely to be assigned to the surgical treatment group rather than the medical treatment group. This is because high-grade IVH is contraindicated for medical treatment. In this study, high grade IVH was not increased in the surgical group (16.4\% in the medical group vs. $21.1 \%$ in the surgical group). However, in other studies, ${ }^{25,26}$ IVH was increased after PDA ligation. Those studies have suggested that rapid increase of previously low cerebral perfusion can cause reperfusion injury and IVH. ${ }^{25,26}$ Recently, clinicians have suggested that PDA should be treated if cerebral perfusion and oxygenation is subnormal. ${ }^{27-29}$

PDA has been reported to be associated with an increased risk of severe BPD. ${ }^{26}$ In the present study, moderate to severe BPD was more frequent in the surgical group than in the medical group (21.7\% in the medical group vs. $36.7 \%$ in the surgical group). However, the difference between the two groups was not statistically significant. Surgical treatment of PDA showed increased risk of BPD compared to medical treatment (OR: 4.0 for surgical treatment and 2.1 for medical treatment compared to no treatment). ${ }^{26} \mathrm{PDA}$ ligation has been suggested as a risk factor for the development of BPD previously. ${ }^{30}$ However, the causal role of HSDA in BPD development remains controversial, although numerous studies support an association of prolonged HSDA with BPD. ${ }^{31}$ High frequency ventilation is known to decrease shunt flow of HSDA by decreasing left to right shunt with higher mean airway pressure. ${ }^{32}$ In the present study, the duration of high frequency ventilation was longer in the surgical treatment group. However, it became similar after adjusting for GA and birth weight. Further study is needed to clarify the association between mean airway pressure of high frequency ventilation (not the duration of high frequency ventilation) and PDA.

In this study, the risk factor of surgical ligation was lower GA as expected. Lower GA has been reported to be a significant risk factor for failure of medical treatment. ${ }^{33}$ In preterm infants with GA of 26-29 weeks, antenatal steroids therapy and histologically confirmed chorioamnionitis decreased symptomatic PDA. ${ }^{33}$ However, in the present study, antenatal steroids therapy or histologically confirmed chorioamnionitis was not significantly related to surgical ligation of PDA.

This study has some limitations. Because of its retrospective nature, survival bias and selection bias could be confounding factors. Infants who were confirmed to have PDA, but it was unknown whether their PDA was closed or not later (who were dead or transferred to another hospital) were excluded. If they survived or were not transferred to another hospital, they might be enrolled in a surgical group. Medical treatment was contraindicated for high-grade IVH ( $\geq$ grade 3 ) or NEC ( $\geq$ stage 2 ). Thus, rather severe infants with these morbidities might be allocated to the surgical group. A follow-up study is needed to determine the long-term neurodevelopmental outcome of those surgically treated infants. 
In conclusion, infants in the surgical group had younger GA and smaller birth weight compared to those in the medical group. The risk factor of surgical ligation was lower GA. Surgical group needed more ventilation, although postnatal day of PDA closure was not delayed. These results imply that surgical treatment related adverse effects should be considered. Surgical treatment of PDA is inevitable in certain cases with large ductal shunt and severe respiratory failure. Clinicians should weigh the benefit of ductal closure, complications associated with surgical treatment, and the possibility of spontaneous closure. There must be unit variations of treatment for HSDA. Cerebral oxygenation associated with HSDA and long-term neurodevelopmental outcomes also should be considered when establishing treatment guidelines for HSDA in preterm infants.

\section{Conflict of interest}

No potential conflict of interest relevant to this article was reported.

\section{References}

1) Rolland A, Shankar-Aguilera S, Diomandé D, Zupan-Simunek V, Boileau P. Natural evolution of patent ductus arteriosus in the extremely preterm infant. Arch Dis Child Fetal Neonatal Ed 2015;100:F55-8.

2) Lee JA. Practice for preterm patent ductus arteriosus; focusing on the hemodynamic significance and the impact on the neonatal outcomes. Korean J Pediatr 2019;62:245-51.

3) Prescott S, Keim-Malpass J. Patent ductus arteriosus in the preterm infant: diagnostic and treatment options. Adv Neonatal Care 2017;17:108.

4) Fowlie PW, Davis PG. Prophylactic intravenous indomethacin for preventing mortality and morbidity in preterm infants. Cochrane Database Syst Rev 2002;(3):CD000174.

5) Fowlie PW, Davis PG, McGuire W. Prophylactic intravenous indomethacin for preventing mortality and morbidity in preterm infants. Cochrane Database Syst Rev 2010;(7):CD000174.

6) Jensen EA, Dysart KC, Gantz MG, Carper B, Higgins RD, Keszler M, et al. Association between use of prophylactic indomethacin and the risk for bronchopulmonary dysplasia in extremely preterm infants. J Pediatr 2017;186:34-40.e2.

7) Jensen EA, Foglia EE, Schmidt B. Association between prophylactic indomethacin and death or bronchopulmonary dysplasia: a systematic review and meta-analysis of observational studies. Semin Perinatol 2018;
42:228-34

8) Su BH, Lin HY, Chiu HY, Tsai ML, Chen YT, Lu IC. Therapeutic strategy of patent ductus arteriosus in extremely preterm infants. Pediatr Neonatol 2020;61:133-41.

9) Sung SI, Lee MH, Ahn SY, Chang YS, Park WS. Effect of nonintervention vs oral ibuprofen in patent ductus arteriosus in preterm infants: a randomized clinical trial. JAMA Pediatr 2020;174:755-63.

10) Volpe JJ. Intraventricular hemorrhage in the premature infant--current concepts. Part I. Ann Neurol 1989;25:3-11.

11) International Committee for the Classification of Retinopathy of Prematurity. The International Classification of Retinopathy of Prematurity revisited. Arch Ophthalmol 2005;123:991-9.

12) Kamitsuka MD, Horton MK, Williams MA. The incidence of necrotizing enterocolitis after introducing standardized feeding schedules for infants between 1250 and 2500 grams and less than 35 weeks of gestation. Pediatrics 2000;105:379-84.

13) Jeon GW. Surfactant preparations for preterm infants with respiratory distress syndrome: past, present, and future. Korean J Pediatr 2019;62: 155-61.

14) Jobe AH, Bancalari E. Bronchopulmonary dysplasia. Am J Respir Crit Care Med 2001;163:1723-9.

15) Mashally S, Nield LE, McNamara PJ, Martins FF, El-Khuffash A, Jain A, et al. Late oral acetaminophen versus immediate surgical ligation in preterm infants with persistent large patent ductus arteriosus. J Thorac Cardiovasc Surg 2018;156:1937-44.

16) Han $C$, Jeon GW. Differences in outcomes according to the time of patent ductus arteriosus closure in preterm infants. Neonatal Med 2020; 27:111-7.

17) Ezenwa B, Pena E, Schlegel A, Bapat R, Shepherd EG, Nelin LD. Effects of practice change on outcomes of extremely preterm infants with patent ductus arteriosus. Acta Paediatr 2019;108:88-93.

18) Chang YH, Lee JY, Kim JE, Kim JY, Youn Y, Lee EJ, et al. The Aristotle score predicts mortality after surgery of patent ductus arteriosus in preterm infants. Ann Thorac Surg 2013;96:879-84.

19) Mirea L, Sankaran K, Seshia M, Ohlsson A, Allen AC, Aziz K, et al. Treatment of patent ductus arteriosus and neonatal mortality/morbidities: adjustment for treatment selection bias. J Pediatr 2012;161:689-94.e1.

20) Weisz DE, Mirea L, Resende MHF, Ly L, Church PT, Kelly E, et al. Outcomes of surgical ligation after unsuccessful pharmacotherapy for patent ductus arteriosus in neonates born extremely preterm. J Pediatr 2018; 195:292-6.e3.

21) Weisz DE, Mirea $L$, Rosenberg E, Jang $M$, Ly L, Church PT, et al. Association of patent ductus arteriosus ligation with death or neurodevelopmental impairment among extremely preterm infants. JAMA Pediatr 2017;171: 443-9.

22) Weisz DE, More K, McNamara PJ, Shah PS. PDA ligation and health out comes: a meta-analysis. Pediatrics 2014;133:e1024-46.

23) Lemmers PM, Benders MJ, D'Ascenzo R, Zethof J, Alderliesten T, Kersbergen KJ, et al. Patent ductus arteriosus and brain volume. Pediatrics 2016;137:e20153090.

24) Weisz DE, Giesinger RE. Surgical management of a patent ductus arte- 
riosus: is this still an option? Semin Fetal Neonatal Med 2018;23:255-66.

25) Soleymani S, Khoo MC, Noori S, Seri I. Modeling of neonatal hemodynamics during PDA closure. Conf Proc IEEE Eng Med Biol Soc 2015;2015: 1886-9.

26) Härkin P, Marttila R, Pokka T, Saarela T, Hallman M. Morbidities associated with patent ductus arteriosus in preterm infants. Nationwide cohort study. J Matern Fetal Neonatal Med 2018;31:2576-83.

27) Ledo A, Aguar M, Núñez-Ramiro A, Saénz P, Vento M. Abdominal nearinfrared spectroscopy detects low mesenteric perfusion early in preterm infants with hemodynamic significant ductus arteriosus. Neonatology 2017;112:238-45.

28) Jeon GW. Clinical application of near-infrared spectroscopy in neonates. Neonatal Med 2019;26:121-7.

29) Sung SI. Controversy in the diagnosis and treatment of hemodynamically significant patent ductus arteriosus in preterm infants. Korean J Pediatr 2019:62:410-1.

30) Clyman RI. The role of patent ductus arteriosus and its treatments in the development of bronchopulmonary dysplasia. Semin Perinatol 2013; 37:102-7.

31) Clyman RI. Patent ductus arteriosus, its treatments, and the risks of pulmonary morbidity. Semin Perinatol 2018;42:235-42.

32) Fajardo MF, Claure N, Swaminathan $S$, Sattar S, Vasquez A, D'Ugard C, et al. Effect of positive end-expiratory pressure on ductal shunting and systemic blood flow in preterm infants with patent ductus arteriosus. Neonatology 2014;105:9-13.

33) Lee JA, Sohn JA, Oh S, Choi BM. Perinatal risk factors of symptomatic preterm patent ductus arteriosus and secondary ligation. Pediatr Neonatol 2020;61:439-46. 